Henry Fast, 1758 De Marietta Ave., Apt 1, San Jose, CA 95126

\title{
THE INJECTIVITY OF THE CROFTON TRANSFORM
}

\begin{abstract}
As an extension of a result from $[\mathrm{F}]$ an example is constructed of two different plane curves whose Crofton transforms are almost everywhere finite and equal each other on a metrically dense set.
\end{abstract}

\section{Introduction}

Let $L$ denote the space of all lines in $\mathbb{R}^{2}$ endowed with the natural topology and measure. The reader is referred to $[\mathrm{F}]$ for the definition of this natural topology and measure on $L$ as well as other definitions not in this paper. The result obtained in $[\mathrm{F}]$ (Theorem 10.1) states that within a certain subclass of rectifiable subsets of $\mathbb{R}^{2}$ (which includes in particular rectifiable arcs and curves) a set is retrievable from its Crofton transform uniquely modulo a set of linear measure zero, provided that the transform is given on a subset of full measure of $L$.

One may think that a set might be retrievable from its Crofton transform even under a weaker assumption about the subset of $L$ on which the transform is given, namely that the set is metrically dense. (In which case the almost everywhere finiteness of the Crofton Transform would have to be introduced as an extra assumption since it wouldn't be implied by the rectifiability of its generating set.) The example below shows that the assumption of full measure in the quoted result can't be weakened to metrically dense.

Example 1.1 There are two distinct rectifiable closed Jordan curves $\sigma^{j}, j=$ 1,2 in $\mathbb{R}^{2}$ with $N_{\sigma^{j}}<\infty|\cdot|_{2}$ almost everywhere on $L$ for $j=1,2$ and for which $\left\{\ell \in L: N_{\sigma^{1}}(\ell)=N_{\sigma^{2}}(\ell)\right\}$ is $|\cdot|_{2}$ metrically dense in $L$.

Key Words: Crofton transform, inversion, injectivity, rectifiable curve

Mathematical Reviews subject classification: Primary: 28A75

Received by the editors August 13, 1995 


\section{Definitions and Notation}

We use the notation introduced in [F]. (In particular we would like to remind the reader that the value of the Crofton transform $N_{E}(\ell)$ of an $E \subset \mathbb{R}^{2}$ is the number (finite or $\infty$ ) of points of the set $\ell \cap E$.) In addition we use the following:

1. $O p(L)$ denotes the class of open subsets of $L$.

2. $\mathcal{V}(L)$ denotes the base of neighborhoods in the topology on $L$ of the form: $\left\{\ell \in L: \delta\left(z_{j}, \ell\right)<\epsilon\right\}$ for $j=1,2$, where $\epsilon>0$ and $z_{j}$ are two distinct points.

3. For $S \subset \mathbb{R}^{2}, \operatorname{Conv}(S)$ denotes the closed convex hull of $S$.

4. For $S \subset \mathbb{R}^{2}, \mathcal{V}(L \mid S$, const $)=\left\{V \in \mathcal{V}(L): N_{S}\right.$ is constant on $\left.V\right\}$ (the S-neighborhoods of constancy).

5. Let $\mathcal{P}$ denote the class of simple closed polygons.

6. For $S \in P$ let $\mathcal{H}(S)=\left\{\phi: S \rightarrow S^{\prime}: \phi\right.$ is a homeomorphism and $\left.S^{\prime} \in \mathcal{P}\right\}$. In particular $\phi_{S}^{I}$ denotes the identity mapping of $S$ onto itself.

7. For any $\sigma=\left(\sigma^{1}, \sigma^{2}\right) \in 2^{\mathbb{R}^{2}} \times 2^{\mathbb{R}^{2}}$ let $E(\sigma)=\left\{\ell \in L: N_{\sigma^{1}}(\ell)=N_{\sigma^{2}}(\ell)\right\}$.

Note that for $S \in \mathcal{P}$

(a) since the set of vertices of $S$ is finite, every $G \in O p(L)$ contains a neighborhood of constancy of $N_{S}$

(b) $N_{S}<\infty$ except on a finite subset of $L$ for $S \in \mathcal{P}$.

Definition 2.1 Let $\epsilon>0, S \in \mathcal{P}, \mathcal{V} \in \mathcal{V}(L)$ and let $m$ be a nonnegative even integer. A mapping $\phi \in \mathcal{H}(\mathcal{S})$ is an $(\epsilon-m)$-upgrader of $S$ over a neighborhood $V$ means:

(u1) $V \subset\left\{\ell \in L: N_{\phi(S)}(\ell)-N_{S}(\ell)=m\right\}$

(u2) $\operatorname{Conv}(S)=\operatorname{Conv}(\phi(S))$

(из) $\max \{|\phi(z)-z|: z \in S\}<\epsilon$

(u4) $\operatorname{diam}(\phi(S) \nabla S)<\epsilon$

(u5) $|S|_{1} \leq|\phi(S)|_{1} \leq|S|_{1}+\epsilon$. 
(u6) $N_{S}(\ell) \leq N_{\phi(S)}(\ell)$ when $N_{S}(\ell)<\infty$.

Note that for $m=0, \phi_{S}^{I}$ is a trivial $(\epsilon-\mathrm{m})$ upgrader of $S$.

Definition 2.2 Let $\epsilon>0$ and let $\sigma^{j} \in \mathcal{P}$ for $j=1,2$. An ordered pair $\psi=$ $\left(\psi^{1}, \psi^{2}\right) \in \mathcal{H}\left(\sigma^{1}\right) \times \mathcal{H}\left(\sigma^{2}\right)$ is an $\epsilon$-equalizer of the ordered pair $\sigma=\left(\sigma^{1}, \sigma^{2}\right)$ over a neighborhood $V \in \mathcal{V}(L)$ means

(e1) $V \subset E(\psi(\sigma))$

(e2) $\max \left\{\left|\psi^{j}(z)-z\right|: z \in \sigma^{j}\right\}<\epsilon$ for $j=1,2$.

Note that if $V \subset E(\sigma)$, then $\left(\phi_{\sigma^{1}}^{I}, \phi_{\sigma^{2}}^{I}\right)$ is a (trivial) $\epsilon$-equalizer of $\sigma$ over $V$ for every $\epsilon>0$.

\section{Lemmas}

The following lemmas form the basis for the forthcoming construction.

Lemma 3.1 Let $m$ be a nonnegative even integer, $S \in \mathcal{P}$ and $V \in \mathcal{V}(L)$ with $V \cap\left\{\ell \in L: N_{S}(\ell)>0\right\} \neq \emptyset$. Then for $\epsilon>0$ sufficiently small there are $V^{\prime} \subset V$ and $V^{\prime} \in \mathcal{V}(L, S$, const $)$ and an $(\epsilon-m)$-upgrader $\phi$ of $S$ over $V^{\prime}$.

Proof. Select $\ell \in V$ which does not meet any of the vertices of $S$. Let $V^{*} \in \mathcal{V}(L \mid S$, const $)$ such that $\ell \in V^{*} \subset V$. Since for $m=0$ clearly $\phi_{S}^{I}$ is a $(\epsilon-0)$ upgrader of $S$ over $V^{*}$, assume $m>0$. Select an arbitrary point $z \in S \cap \ell$ and let $\epsilon>0$ be sufficiently small so that the $\epsilon$ - disk about $z$ meets $S$ on a straight-line segment. Call this segment $J$. For $\epsilon$ sufficiently small we also have $\epsilon<\delta(z, S \backslash J)$. Select two distinct points $z_{j} \in \operatorname{int} \operatorname{Conv}(S) \cap \ell$ for $j=1,2$ with $\left|z_{2}-z_{1}\right|<\epsilon$ and $\left|z_{j}-z\right|<\epsilon, j=1,2$ and connect them by a polygonal $\operatorname{arc} Z$ (a 'zigzag') having the following properties:

(a) $Z \subset \operatorname{int} \operatorname{Conv}(S)$

(b) consecutive vertices of $Z$ different from its endpoints $z^{j}$ are on opposite sides of $\ell$ all at the same distance from $\ell$

(c) $|Z|_{1}<2 \epsilon$.

Rotate $Z$ by an angle $\alpha$ about the midpoint of $z^{1}$ and $z^{2}$ situated on $\ell$ to a new position $Z_{\alpha}$ choosing $\alpha$ sufficiently small to preserve the inclusion $Z_{\alpha} \subset$ int $\operatorname{Conv}(S)$ and to keep the vertices of the zigzag from crossing $\ell$ in the process. Connect the two endpoints of $Z_{\alpha}$, now on opposite sides of $\ell$, with the two endpoints of $J$ using two polygons $I_{j}$ for $j=1,2$ satisfying the conditions: 
(a') $I_{j} \cap \ell=\emptyset$ for $j=1,2$

(b') $I_{j} \subset \operatorname{Conv}(S)$ for $j=1,2$

(c') $\left|I_{j}\right|_{1}<2 \epsilon$ for $j=1,2$.

The polygon $J^{\prime}=I^{1} \cup Z_{\alpha} \cup I^{2}$ joins the endpoints of $J$ and satisfies $J^{\prime} \subset \operatorname{Conv}(S)$ and $\left|J^{\prime}\right|_{1}<6 \epsilon$. Through appropriate choices of the number of vertices of $Z$ and of $\alpha N_{J^{\prime}}(\ell)=m+1$. Since $N_{J}(\ell)=1$, the difference $N_{J^{\prime}}(\ell)-N_{J}(\ell)=m$. Define $\phi \in \mathcal{H}(S)$ as the identity on $S \backslash J$ and as a homeomorphism of $J$ onto $J^{\prime}$. We have $\phi(S)=S^{\prime}$ and $N_{S^{\prime}}(\ell)-N_{S}(\ell)=$ $N_{J^{\prime}}(\ell)-N_{S}(\ell)=m$.

Note that in the above construction $\ell$ does not meet any of the vertices of $S^{\prime}$. Thus there is a $V^{\prime} \subset V^{*}$ with $V^{\prime} \in V\left(L \mid S^{\prime}\right.$, const $)$ such that $\ell \in V^{\prime}$ and $N_{\phi(S)}\left(\ell^{\prime}\right)-N_{S}\left(\ell^{\prime}\right)=m$ for $\ell^{\prime} \in V^{\prime}$. This shows that (u1) holds for $\phi$. It is easy to check that the remaining five upgrader defining properties are satisfied as well.

Lemma 3.2 Let $\sigma=\left(\sigma^{1}, \sigma^{2}\right) \in \mathcal{P} \times \mathcal{P}$ and let $G \in O p(L)$ and let there be $a \ell \in G$ for which $N_{\sigma^{1}}(\ell), N_{\sigma^{2}}(\ell)>0$. For $\epsilon>0$ sufficiently small there is a $V \subset G$ with $V \in \mathcal{V}(L)$ and an $\epsilon$-equalizer of $\sigma$ over $V$. Moreover, this equalizer has one of the two forms: $\psi=\left(\phi, \phi_{\sigma^{2}}^{I}\right)$ where $\phi \in \mathcal{H}\left(\sigma^{1}\right)$ or $\psi=\left(\phi_{\sigma^{1}}^{I}, \phi\right)$ where $\phi \in \mathcal{H}\left(\sigma^{2}\right)$.

Proof. When $G \cap E(\sigma) \neq \emptyset$ select any $V$ such that $V \subset G \cap E(\sigma)$ with $V \in \mathcal{V}(L)$. Then $\psi=\left(\phi_{S^{1}}^{I}, \phi_{S^{2}}^{I}\right)$ is a (trivial) equalizer satisfying the conclusion of the Lemma.

If $G \cap E(\sigma)=\emptyset$, select a $V$ such that $V \subset G$ with $V \in \mathcal{V}(L)$ and a $\ell \in V$ such that no vertices of either $\sigma^{1}$ nor $\sigma^{2}$ are on $\ell$. Assume for definiteness that $N_{\sigma^{2}}(\ell)-N_{\sigma^{1}}(\ell)=m>0$. Use as $\phi^{1}$ an $\left(\epsilon\right.$-m)-upgrader $\phi$ of $\sigma^{1}$ over $V$ as produced in Lemma 3.1. Then $\psi=\left(\phi, \phi_{\sigma^{2}}^{I}\right)$ satisfies both (e1) and (e2). Thus it is an equalizer, as claimed. The case $m<0$ yields the alternate form.

Lemma 3.3 Let $D \subset \mathbb{R}^{2}$ be closed. Then $\left|\left\{\ell: N_{D}>0\right\}\right|_{2} \leq \operatorname{diam}(D)$.

Proof. Let $\chi$ denote the characteristic function of $\left\{\ell: N_{D}>0\right\}$ and $L(\nu)$ denote the subset of $L$ consisting of lines in direction $\nu$ where $\nu \in C$ (that is, $|\nu|=1$. Then

$$
\left|\left\{\ell: N_{D}>0\right\}\right|_{2}=\iint_{L}|\chi(\ell)||d(\ell)|_{2}=\int_{C}|d \nu|_{1} \int_{L(\nu)}|\chi(\ell)||d(\ell)|_{1} \leq
$$




$$
\operatorname{diam}(D) \int_{C}|d \nu|_{1}=\operatorname{diam}(D)
$$

We assume here that the measure on $C$ is normalized; that is, $\int|d \nu|_{1}=1$.

Lemma 3.4 Let $\sigma=\left(\sigma^{1}, \sigma^{2}\right) \in \mathcal{P} \times \mathcal{P}$ such that $\operatorname{Conv}\left(\sigma^{1}\right)=\operatorname{Conv}\left(\sigma^{2}\right)$ and let $G \in O p(L)$. For $\epsilon>0$ sufficiently small there are $V \subset G$ with $V \in \mathcal{V}(L)$ and an $\epsilon$-equalizer $\psi$ of $\sigma$ over $V$ such that $|E(\sigma) \backslash E(\psi(\sigma))|_{2}<\epsilon$.

Proof. If there is an $V \subset G$ with $V \in \mathcal{V}(L)$ having the property that $V \subset E(\sigma)$ then the trivial equalizer $\left.\psi=\phi_{\sigma^{1}}^{I}, \phi_{\sigma^{2}}^{I}\right)$ over that $V$ will do. That will be the case when $G \cap \operatorname{Conv}\left(\sigma^{1}\right)=\emptyset$ and in this case $G \cap \operatorname{Conv}\left(\sigma^{2}\right)=\emptyset$ as well.

Should $G$ be such that no such $V$ exists, select an arbitrary $V \subset G$ with $V \in$ $\mathcal{V}\left(L \mid \sigma^{1}\right.$ const $) \cap \mathcal{V}\left(L \mid \sigma^{2}\right.$, const $)$. In this case we also require $N_{\sigma^{1}}(\ell), N_{\sigma^{2}}(\ell)>0$ for every $\ell \in V$.

Without loss of generality assume that $m=N_{\sigma^{2}}-N_{\sigma^{1}}>0$ over $V$. Let $\phi$ be an $(\epsilon-m)$-upgrader of $\sigma^{1}$ over $V$. Set $\psi=\left(\phi, \phi_{\sigma^{2}}^{I}\right)$. Then $\psi$ is an $\epsilon$-equalizer of $\sigma$ over $V$ and is of one of the two forms as provided by Lemma 3.2. The deformation produced in $\sigma^{1}$ by $\psi$ is confined to an $\epsilon$-disk about one of its points. By Lemma 3.3 every $\ell^{\prime} \in E(\sigma) \backslash E(\psi(\sigma))$ meeting such disk belongs to a subset of $L$ whose $|\cdot|_{2}$-measure is at most $\epsilon$.

Lemma 3.5 Let $r \in(0,1)$ and $G \in O p(L)$. The equalizer in Lemma 3.4 may be chosen such that $|G \cap E(\sigma) \cap E(\psi(\sigma))|_{2} \geq r \cdot|G \cap E(\sigma)|_{2}$.

Proof. This is an immediate consequence of Lemmas 3.3 and 3.4 when $\epsilon$ is taken sufficiently small.

Definition 3.1 A quintuple $(\sigma, W, \mathcal{E}, \Psi, \hat{\Psi})$ is called a chain, means:

(a) $\sigma=\left(\sigma^{1}, \sigma^{2}\right) \in \mathcal{P} \times \mathcal{P}$ with $\operatorname{Conv}\left(\sigma^{1}\right)=\operatorname{Conv}\left(\sigma^{2}\right)$,

(b) $W=\left\{V_{n}\right\}_{n=1}^{\infty}: V_{n} \in \mathcal{V}(L)$

(c) $\mathcal{E}=\left\{\epsilon_{n}\right\}_{n=1}^{\infty}: \epsilon_{n}>0$.

(d) $\Psi=\left\{\psi_{n}\right\}_{n=1}^{\infty}$

(e) $\hat{\Psi}=\left\{\hat{\psi}_{n}\right\}_{n=1}^{\infty}$

satisfying

(1) $\hat{\psi}_{n}=\left(\hat{\psi}_{n}^{1}, \hat{\psi}_{n}^{2}\right)=\psi_{n} \circ \cdots \circ \psi_{1}=\left(\psi_{n}^{1} \circ \cdots \circ \psi_{1}^{1}, \psi_{n}^{2} \circ \cdots \circ \psi_{1}^{2}\right)$, and $\hat{\psi}_{0}(\sigma)=\sigma$, 
(2) $\psi_{n+1}$ is an $\epsilon_{n}$-equalizer of $\psi_{n}(\sigma)$ over $V_{n}$

(3) $V_{n+1} \in \mathcal{V}\left(L \mid \psi_{n}(\sigma)\right.$, const $)$,

(4) $\operatorname{Conv} \hat{\psi}_{n}^{j}\left(\sigma^{j}\right) ; j=1,2$ are all equal.

Lemma 3.6 Let $\sigma=\left(\sigma^{1}, \sigma^{2}\right)$ be a pair of polygons from $\cap P$ and let $\left\{G_{n}\right\}_{n=1}^{\infty}$ be a countable base of $O p(L)$. There is a chain $(\sigma, W, \mathcal{E}, \Psi, \hat{\Psi})$ with the property: for every natural $m$ and $1 \leq k \leq m$

$$
\left|G_{k} \cap \bigcap_{i=k-1}^{m} E\left(\hat{\psi}_{i}(\sigma)\right)\right|_{2} \geq \prod_{i=1}^{m-k+1}\left(1-\frac{1}{2^{i}}\right)\left|G_{k} \cap E\left(\psi_{1}(\sigma)\right)\right|_{2} .
$$

Proof. Assume inductively that for a given $m$ we already have a $m$-partial chain in which $\left(^{*}\right)$ holds for $k=1, \ldots, m$. When $m=1$ the inequalities $(*)$ are reduced to $\left|G_{1} \cap E(\sigma) \cap E\left(\psi_{1}(\sigma)\right)\right|_{2} \geq \frac{1}{2}\left|G_{1} \cap E(\sigma)\right|_{2}$.

Lemma 3.5 applied to the open set $G_{m+1}$ and $\hat{\psi}_{m}(\sigma)$ as the primary pair of polygons yields the existence of a $V_{m+1} \subset G_{m+1}$ with $V \in \mathcal{V}(L)$, of an $\epsilon_{m+1}>0$ and of an $\epsilon_{m+1}$-equalizer $\psi_{m+1}$ of the pair $\hat{\psi}_{m}(\sigma)$ over $V_{m+1}$ such that

$$
\begin{gathered}
\left|G_{m+1} \cap E\left(\hat{\psi}_{m}(\sigma)\right) \cap E\left(\psi_{m+1} \circ \hat{\psi}_{m}(\sigma)\right)\right|_{2}=\left|G_{m} \cap E\left(\hat{\psi}_{m}(\sigma)\right) \cap E\left(\hat{\psi}_{m+1}(\sigma)\right)\right|_{2} \geq \\
\frac{1}{2}\left|G_{m+1} \cap E\left(\hat{\psi}_{m+1}(\sigma)\right)\right|_{2}
\end{gathered}
$$

and this is the $m+1$-st of the inequalities of $\left(^{*}\right)$ for $m+1$.

Note that by the Lemma 3.5 the same $\epsilon_{m+1}$ when selected sufficiently small, yields along with its corresponding $\psi_{m+1}$ in addition to the above

$$
\begin{gathered}
\left|G_{k} \cap \bigcap_{i=k-1}^{m} E\left(\hat{\psi}_{i}(\sigma)\right) \cap E\left(\psi_{m+1}(\sigma)\right)\right|_{2}=\left|G_{k} \cap \bigcap_{i=k-1}^{m+1} E\left(\hat{\psi}_{i}(\sigma)\right)\right|_{2} \geq \\
\left(1-\frac{1}{2^{m-k+2}}\right)\left|G_{k} \cap \bigcap_{i=k-1}^{m} E\left(\hat{\psi}_{i}(\sigma)\right)\right|_{2} \geq \prod_{i=1}^{m-k+2}\left(1-\frac{1}{2^{i}}\right)\left|G_{k} \cap E\left(\psi_{1}(\sigma)\right)\right|_{2},
\end{gathered}
$$

for all the $k=1, \ldots, m$ simultaneously, in short,

$$
\left|G_{k} \cap \bigcap_{i=k-1}^{m} E\left(\hat{\psi}_{i}(\sigma)\right)\right|_{2} \geq \prod_{i=1}^{(m+1)-k-1}\left(1-\frac{1}{2^{i}}\right)\left|G_{k} \cap E\left(\psi_{1}(\sigma)\right)\right|_{2},
$$

which is the $k^{\prime}$ th inequality of $(*)$ with $m+1$ for values $1 \leq k \leq m$. 
Lemma 3.7 Under the condition of the previous Lemma there exists a chain $(\sigma, W, E, \Phi, \hat{\Psi})$ such that the set

$$
E=\bigcup_{n=1}^{\infty} \bigcap_{k=n}^{\infty} E\left(\hat{\psi}_{k}(\sigma)\right)
$$

is $|\cdot|_{2}$ metrically dense in $L$.

Proof. Passing from a fixed $m$ to the limit as $k \rightarrow \infty$ in $\left(^{*}\right)$ and considering that $\prod_{i=1}^{\infty}\left(1-\frac{1}{2^{i}}\right)>0,\left|G_{m} \cap E\left(\hat{\psi}_{m}(\sigma)\right)\right|_{2}>\left|V_{m}\right|_{2}>0$ and $V_{m} \subset G_{n} \cap$ $E\left(\psi_{n}(\sigma)\right)$, we obtain

$$
\left|G_{m} \cap \bigcap_{i=m}^{\infty} E\left(\hat{\psi}_{i}(\sigma)\right)\right|_{2} \geq \prod_{i=1}^{\infty}\left(1-\frac{1}{2^{i}}\right)\left|G_{m} \cap E\left(\hat{\psi}_{m}(\sigma)\right)\right|_{2}>0
$$

which ends the proof.

\section{The Main Result}

Now we are ready to establish the example mentioned in Section 1. Let $\sigma_{0}^{1}$ be the boundary of the unit square and let $\sigma_{0}^{2}$ be the simple closed octagon obtained from $\sigma_{0}^{1}$ by bending each of its four edges inward into the square. We have $\sigma_{0}^{j} \in \mathcal{P}$ for $j=1,2$ and $\operatorname{Conv}\left(\sigma_{0}^{1}\right)=\operatorname{Conv}\left(\sigma_{0}^{2}\right)$. Let $\left(\sigma_{0}, W, E, \Psi, \hat{\Psi}\right)$ be a chain with $\sum_{n=1}^{\infty} \epsilon_{n}<\infty$. Then $\lim _{n \rightarrow \infty} \hat{\psi}_{n}=f$ uniformly on $\sigma_{0}$, where $f=\left(f_{1}, f_{2}\right): \sigma_{0} \rightarrow \mathbb{R}^{2} \times \mathbb{R}^{2}$. The two continuous mappings $f_{j}: \sigma_{0}^{j} \rightarrow \mathbb{R}^{2}$ produce two closed Jordan curves $\sigma^{j}=f_{j}\left(\sigma_{0}^{j}\right)$ in $\mathbb{R}^{2}$ for $j=1,2$

which (when each $\epsilon_{n} ; n=1,2, \ldots$ is sufficiently small) are evidently distinct. Condition (u5) guarantees their rectifiability.

By (u6) we have $N_{\psi_{n}^{j}\left(\sigma^{1}\right)} \leq N_{\psi_{n+1}^{j}\left(\sigma^{2}\right)}$ for $j=1,2$ and $n=1,2, \ldots$. Since each $\sigma^{j}$ is rectifiable, their Crofton Transforms are finite $|\cdot|_{2}$ almost everywhere on $L$. This implies that $|\cdot|_{2^{-}}$-almost every sequence $\left[N_{\hat{\psi}_{n}^{j}\left(\sigma^{j}\right)}(\ell)\right]_{n=1}^{\infty}$ attains its limit for a certain value $n_{j}=n_{j}(\ell)$ of the subscript. For a given $\ell \in E$, by Lemma 3.8 , there is an $m \in \mathbf{N}$ such that $N_{\hat{\psi}_{n}^{1}\left(\sigma^{1}\right)}(\ell)=N_{\hat{\psi}_{n}^{2}\left(\sigma^{2}\right)}(\ell)$ for $n \geq m$, which implies that $N_{\sigma^{1}}(\ell)=N_{\sigma^{2}}(\ell)$.

\section{References}

[F] H. Fast, Inversion of the Crofton Transform for sets in the plane, Real Analysis Exchange, 19 (1) (1993-94), 59-80. 